\title{
Insomnia Symptoms and Well-Being: Longitudinal Follow-Up
}

\author{
Cynthia W. Karlson, Matthew W. Gallagher, Christy A. Olson, and Nancy A. Hamilton \\ University of Kansas
}

\begin{abstract}
Objective: Most Americans have occasional problems with symptoms of insomnia. Insomnia symptoms have been linked to psychological distress, but few studies have examined the relationship between insomnia symptoms and well-being. The purpose of the present study was to assess the relationship between insomnia symptoms reported in a 10-year longitudinal study and the dimensions of subjective well-being and eudaimonic well-being, adjusting for the potential confounds of age, gender, and comorbid physical illness. Method: The data for the present study came from the National Survey of Midlife Development in the United States. Participants were 4,014 community dwelling adults ( $M$ age $=$ 56.27 years, $S D=12.4 ; 55.4 \%$ female; $91.6 \%$ White). Results: After adjusting for demographic characteristics and a wide range of chronic physical health conditions, we found that insomnia symptoms had a significant relationship with both subjective and eudaimonic well-being. Furthermore, the report of insomnia symptoms at 2 time points 10 years apart was found to have an additional impact on subjective and eudaimonic well-being. Conclusions: Results of this study suggest that insomnia symptoms have a strong relationship to individuals enjoying life and perceiving that one has a meaningful life. In addition, these data suggest that the experience of recurrent insomnia symptoms at 2 time points is particularly detrimental to one's well-being.
\end{abstract}

Keywords: insomnia symptoms, sleep, subjective well-being, eudaimonic well-being, longitudinal

Only approximately $7 \%$ of the general population meet criteria for chronic insomnia (difficulty with initiating and/or maintaining sleep, lasting at least 1 month), but another $22 \%$ of the population report current "trouble sleeping" or symptoms of insomnia (Bixler, Vgontzas, Lin, Vela-Bueno, \& Kales, 2002). Past research suggests that chronic insomnia may be related to physical and psychological illness (Léger, Scheuermaier, Philip, Paillard, \& Guilleminault, 2001; Philip et al., 2006; Zammit, Weiner, Damato, Sillup, \& McMillan, 1999). However, less is known about the impact of more common recurring symptoms of insomnia on an individual's well-being (e.g., positive affect and purpose in life). Using the latest Midlife Development in the United States (MIDUS)-II data set, the current study followed up our work with the MIDUS-I data that documented a cross-sectional relationship between symptoms of insomnia and well-being. Specifically, we examined the longitudinal relationship between recurring insomnia symptoms at two time points 10 years apart and well-being.

The relationship between insomnia symptoms and physical and psychological illness is well documented. Medical conditions commonly associated with physical discomfort or worry, such as obesity, chronic pain, heart disease, depression, and memory impairment (e.g., reduced ability to consolidate memories), have been found to correlate with symptoms of insomnia (e.g., Foley, Ancoli-Israel, Britz, \& Walsh, 2004; Nissen et al., 2011; Ohayon,

This article was published Online First July 2, 2012

Cynthia W. Karlson, Matthew W. Gallagher, Christy A. Olson, and Nancy A. Hamilton, Department of Psychology, University of Kansas.

Correspondence concerning this article should be addressed to Cynthia W. Karlson, Department of Psychiatry, University of Mississippi Medical Center, 2500 North State Street, Jackson, MS 39216. E-mail: ckarlson@umc.edu
2005). Although these cross-sectional relationships between insomnia symptoms and other disorders are important to examine, there are at least two issues that are not well addressed with correlational data. First, as with all correlational data, the direction of the relationship is ambiguous. Second, these data do not distinguish between individuals who have transient or recurring symptoms of insomnia and those who have chronic insomnia.

There are likely to be far different physical and mental health consequences for people with transient or recurring symptoms of insomnia compared with those who have chronic insomnia. However, teasing apart differences in time course and severity can be challenging. In several research studies, insomnia symptoms have been found to have a cross-sectional relationship with psychological problems such as anxiety, depression, suicidal ideation, and substance abuse (Carli et al., 2011; Ford \& Kamerow, 1989; Redeker et al., 2010). Furthermore, insomnia symptoms have been found to be predictive of anxiety disorders, depressive symptoms, and other psychiatric disorders 1 to 3 years later (Ford \& Kamerow, 1989; Yokoyama et al., 2010). These data clearly suggest that insomnia is a correlate as well as a predictor of psychological disorders.

Chronic insomnia is typically operationalized by taking "snapshots" of sleep problems at multiple time points (Breslau, Roth, Rosenthal, \& Andreski, 1996; Ford \& Kamerow, 1989; Yokoyama et al., 2010). For instance, one longitudinal epidemiological study measured sleep complaints at two time points separated by a 3.5-year interval (Breslau et al., 1996). Almost half of respondents who reported clinically significant insomnia (defined as difficulty sleeping for at least 2 weeks' duration) at baseline continued to report clinically significant insomnia at follow-up. Insomnia in this study was correlated with a higher lifetime risk of depression, anxiety, and substance abuse problems, and insomnia at baseline 
predicted new incidents of psychiatric problems 3.5 years later. Such studies suggest that for many people insomnia may be a recurring problem and one that has an adverse effect on an individual's mental health.

Such cross-sectional and longitudinal research suggests that insomnia is related to current and future psychiatric illness, whereas the relationship between insomnia and current and future psychological well-being is less clear. Well-being is a construct that is used to indicate a state of both physical and mental health rather than the absence of pathology. Past research suggests that the absence of well-being may reduce resilience to adversity and thus increase vulnerability to depression and anxiety (Ryff \& Singer, 1996). In addition, there is a large body of literature on the buffering effects of social relationships and perceived support against life stressors and psychological distress (e.g., Beekman et al., 2002; Uchino, Cacioppo, \& Kiecolt-Glaser, 1996). In one of the most clearly articulated and comprehensive models of wellbeing, this concept is defined by two overarching latent constructs: psychological well-being, also known as subjective well-being (SWB), and eudaimonic well-being (EWB; Keyes, Shmotkin, \& Ryff, 2002). EWB is conceptualized as an agentic approach to life ("challenged thriving"), as well as a sense of meaningful existence, and is defined by traits such as autonomy, environmental mastery, personal growth, positive relations with others, purpose in life, and self-acceptance. In contrast, SWB comprises only two components: a subjective sense of satisfaction with life and high levels of positive affect and enjoyment for life. Thus, SWB has been defined as a general sense of enjoying and being satisfied with one's life, whereas EWB has been defined a general sense of meaning for one's life.

Given the adverse relationship of insomnia symptoms on psychiatric illness over time, it is likely that insomnia symptoms are also related to an individual's sense of well-being over time. However, the extent and trajectory of this relationship is yet unclear. Two known studies have documented cross-sectional relationships between insomnia symptoms and well-being. The first study, making use of the Whitehall II epidemiological sample, illustrated that sleep problems were inversely correlated with both positive affect and EWB (Steptoe, O'Donnell, Marmot, \& Wardle, 2008). Moreover, analyses showed that the relationship between sleep and indicators of psychological dysfunction (i.e., psychological distress, social isolation, negative social relationships, and financial strain) was attenuated after controlling for indices of psychological well-being. Taking a similar approach and making use of the MIDUS-I data, Hamilton and colleagues (2007) documented that insomnia symptoms were correlated with lower levels of psychological well-being. In this study, the relationship between sleep problems and well-being remained significant after controlling for the presence of a wide variety of self-reported physical illnesses, anxiety, and depression. These studies indicate that the relationship between sleep problems and well-being is at least partially independent of physical and psychological problems. Thus, it is important to examine the longitudinal relationship between sleep problems and well-being while controlling for the presence of physical and psychological illness.

The present study examined the 10-year trajectory of the relationship between insomnia symptoms and dimensions of wellbeing, adjusting for the potential confounds of comorbid physical and psychological illness. To obtain a relatively large sample of adult community residents, we relied on data collected as part of the national MIDUS project. Consistent with prior research and the theory that adequate sleep is related to good health (agentic activity) and vigor (related to mood; Adam \& Oswald, 1977), we first hypothesized that insomnia symptoms reported during the MIDUS-II sample (Time 2) would be associated with diminished well-being at Time 2 after covarying for the influence of demographic characteristics and comorbid physical health conditions. We next examined whether insomnia symptoms reported during the MIDUS-I sample (Time 1) predicted future well-being 10 years later at Time 2. Finally, we expanded the model to address the question of whether recurring insomnia symptoms experienced at both Time 1 and Time 2 were related to well-being above that of current insomnia symptoms at Time 2 .

\section{Method}

\section{Procedure}

The data for the present study came from the MIDUS project conducted by the John D. and Catherine T. MacArthur Foundation's Research Network on Successful Midlife Development. The MIDUS project was a multidisciplinary investigation of the factors related to physical and psychological adjustment in American adults. Data for MIDUS-I were collected in 1994-1995 from a national sample of adults between the ages of 20 and 74 years using a random digit dialing procedure. The response rate for the MIDUS-I random digit dialing telephone interview was $70 \%$, with $86.3 \%$ of these individuals (yielding a $60.8 \%$ overall response rate) completing the self-report questionnaires that included the measures of sleep and well-being. Data for MIDUS-II were collected from the same sample of adults 9-10 years later in 2004-2005. The longitudinal response rate across both assessments and adjusted for mortality was $75 \%$. A detailed description of data collection procedures can be found online at the MIDUS Website (http://midus.wisc.edu/) and have been reported by Keyes and colleagues (2002). For the purposes of this project, data were used only from participants who completed the survey at both Time 1 and Time 2. Variables related to sleep, well-being, physical illness, and psychological illness were examined.

\section{Participants}

Of the 4,624 individuals who responded to the original MIDUS-I questions assessing frequency of insomnia symptoms, 4,014 individuals provided enough information during MIDUS-II to be included in final analyses. Table 1 provides select demographic characteristics of these 4,014 participants. Participant age at MIDUS-II ranged from 30 to 85 years. Participants identified themselves as $91.6 \%$ White, $3.7 \%$ Black, $1.5 \%$ Native American, $0.5 \%$ Asian, and $2.3 \%$ other. In addition, $71.1 \%$ were married, $13.8 \%$ of participants were divorced or separated, $7.6 \%$ were widowed, and $7.5 \%$ had never married. The median reported family income was $\$ 57,500$ per year, ranging from $\$ 0$ to $\$ 300,000$ per year. Only $6.8 \%$ of participants failed to complete high school, whereas $28.1 \%$ had graduated from high school or obtained their general education development high school equivalency; $21.6 \%$ had attended some college; $27.3 \%$ had obtained a 2-year or 4-year 
Table 1

Select Demographic and Descriptive Statistics by Participant Gender in MIDUS-II

\begin{tabular}{|c|c|c|c|}
\hline Variable & Men $(n=1,789)$ & Women $(n=2,225)$ & Total $(N=4,014)$ \\
\hline Mean $(S D)$ age (years) & $56.56(12.23)$ & $56.04(12.52)$ & $56.27(12.4)$ \\
\hline White, $\%$ & 92.2 & 91.6 & 91.6 \\
\hline Married, (\%) & 78.7 & 64.9 & 71.1 \\
\hline Mean $(S D)$ family income $(\$)$ & $79,257(61,634)$ & $65,062(58,549)$ & $71,432(60,366)$ \\
\hline \multicolumn{4}{|l|}{ Insomnia symptoms, $\%$} \\
\hline Never & 30.3 & 23.9 & 27.5 \\
\hline One night per month & 18.6 & 15.2 & 16.9 \\
\hline Several nights per month & 17.2 & 17.7 & 17.5 \\
\hline One night per week & 8.4 & 9.4 & 8.9 \\
\hline Several nights per week & 16.8 & 18.7 & 17.8 \\
\hline Almost every night & 8.2 & 14.0 & 11.4 \\
\hline \multicolumn{4}{|l|}{ Recurring insomnia symptoms, \% } \\
\hline Never & 57.1 & 48.8 & 52.7 \\
\hline One night per month & 16.0 & 16.3 & 16.1 \\
\hline Several nights per month & 13.2 & 16.0 & 14.7 \\
\hline One night per week & 4.5 & 4.6 & 4.5 \\
\hline Several nights per week & 5.5 & 8.7 & 7.3 \\
\hline Almost every night & 2.2 & 3.5 & 3.0 \\
\hline
\end{tabular}

college degree; and $16.0 \%$ had attended some graduate school or obtained a graduate degree.

\section{Measures}

Insomnia symptoms. Insomnia symptoms were assessed during both MIDUS-I and MIDUS-II using a single item that asked about the frequency of experienced sleep problems. Participants were asked, "During the past 30 days, how often have you experienced trouble getting to sleep or staying asleep?" To make the data more interpretable, we maintained the original ordinal scale but reverse coded the data so that higher scores reflected a greater frequency of insomnia symptoms: $1=$ not at all, $2=$ once a month, $3=$ several times $a$ month, $4=$ once a week, $5=$ several times a week, and $6=$ almost every day.

We next constructed a recurring insomnia symptoms variable using data from both MIDUS-I and MIDUS-II time points. Participants were assigned a score for recurring insomnia symptoms based on the minimum frequency value of insomnia symptoms at both time points. For example, if a participant rated his/her frequency of insomnia symptoms as 2 at Time 1 and 5 at Time 2, that participant would be assigned a minimum frequency value of 2 for recurring insomnia symptoms. If a participant rated his/her frequency of insomnia symptoms as 6 at Time 1 and 5 at Time 2, the participant would be assigned a minimum frequency value of 5 for recurring insomnia symptoms. This novel method of constructing a recurring insomnia symptoms variable is a more conservative estimate of repeated sleep disturbance compared with the traditional median "yes-no" split used to indicate the existence or lack of sleep problems at multiple time points. In addition, it maintains the original ordinal scale of insomnia symptoms frequency.

Subjective well-being. The three components of SWB used in the MIDUS project included positive affect, negative affect, and life satisfaction. Positive affect and negative affect were assessed using scales specifically developed for the MIDUS project survey (Mroczek \& Kolarz, 1998). Each participant was asked to describe his or her emotional state over the past 30 days by rating 12 adjectives (six positive and six negative) on a 5-point Likert scale.
Negative affect items were reverse coded so that a positive directional relationship indicated better well-being (less negative affect). Scale means were then computed for both positive and negative affect. These scales have previously demonstrated adequate reliability and validity (Mroczek \& Kolarz, 1998). In the current study, MIDUS-II Cronbach's alphas for the positive affect and negative affect scales were .90 and .85 , respectively. Life satisfaction was assessed with a single item at both Time 1 and Time 2. At Time 1, participants rated how satisfied they were with their life on a 4-point Likert scale with responses ranging from $1=$ not at all to $4=a$ lot. At Time 2, participants rated how satisfied they were with their life on an 11-point Likert scale (Cantril, 1965) with responses ranging from $0=$ the worst possible to $10=$ the best possible.

Eudaimonic well-being. The EWB scale assesses six components of Ryff's (1989) model of well-being: autonomy, environmental mastery, personal growth, positive relations with others, purpose in life, and self-acceptance. Each of these six subscales was initially developed to contain 20 items, with original subscale alphas ranging from .86 to .93. Shortened three-item forms of each subscale were developed for the MIDUS survey. Participants responded using a 7-point Likert scale ranging from $1=$ strongly disagree to $7=$ strongly agree In the current study, MIDUS-II Cronbach's alphas for the six subscales ranged from .70 to .84 .

Psychological distress. Scales of depression, generalized anxiety, and panic were created for the MIDUS-I survey to indicate psychological distress based on Diagnostic and Statistical Manual of Mental Disorders (3rd ed. rev.; American Psychiatric Association, 1987) criteria and the World Health Organization (WHO) Composite International Diagnostic Interview-Short Form (Kessler, Andrews, Mroczek, Ustun, \& Wittchen, 1998), which has demonstrated adequate reliability and validity in WHO field trials (Wittchen, 1994). Example items from these scales include, "During two weeks in the past 12 months did you lose interest in most things?" (depression), "How often over the past 12 months were you restless because of your worry?" (generalized 
anxiety), and "When you have attacks, do you have tightness, pain, or discomfort in your chest or stomach?" (panic attack). Scale means for the Depression, Generalized Anxiety, and Panic Attack subscales were specified as the three indicators of psychological distress.

Physical illness. Physical illness variables were selected from the MIDUS-I and MIDUS-II data sets on the basis of their potential effects on sleep or well-being. The following conditions were examined in analyses and had the preface, "In the past 12 months, have you experienced or been treated for any of the following?": heart problems or heart attack; high blood pressure or hypertension; diabetes or high blood sugar; cancer; asthma; tuberculosis; other lung problems; sciatica, lumbago, or recurring backache; migraine headaches; persistent skin trouble; thyroid disease; hay fever; recurring stomach trouble, indigestion, or diarrhea; urinary or bladder problems; being constipated most or all of the time; gall bladder trouble; ulcer; hernia or rupture; piles or hemorrhoids; AIDS or HIV infection; lupus or other autoimmune disorders; multiple sclerosis, epilepsy, or other neurological disorders; stroke; persistent trouble with your gums or mouth; persistent trouble with your teeth; swallowing problems; persistent foot trouble; trouble with varicose veins requiring medical treatment; or alcohol or drug problems.

A physical illness score was coded for both time points using the Medical History Severity Index developed by Benyamini, Leventhal, and Leventhal (1999). This index assigns a numeric value of illness severity to medical conditions. Scores range from 0 to 100 , with higher scores indicating more severe physical illness. If participants had comorbid diagnoses, the highest physical illness score was used.

\section{Data Analytic Plan}

Structural equation modeling (SEM) was used because of its ability to separate common and unique components of variance, thus minimizing the effects of any unreliability due to measure- ment error. For the current study, we focused on examining the effects of insomnia symptoms on three latent variables: SWB, EWB, and psychological distress. For SWB, the three indicators of positive affect, negative affect, and life satisfaction were specified. Three indicators of EWB were created by averaging random pairs of the six subscale scores of Ryff's model to create three parcels, consistent with recommended practice in SEM (Little, Cunningham, Shahar, \& Widaman, 2002). Parcels are advantageous because they are more normally distributed and reliable than the items they comprise and thus conform more closely to the assumptions of maximum likelihood, as well as provide a more parsimonious model. The use of parceling, therefore, reduces measurement bias and improves power. Finally, scale means for depression, generalized anxiety, and panic attack were specified as the three indicators of psychological distress.

We specified three models to better understand the relationship between insomnia symptoms and well-being, while also examining concurrent relationships with psychological distress. The first model was a cross-sectional analysis of the effects of insomnia symptoms on SWB, EWB, and psychological distress in the MIDUS-II data. The second model was a longitudinal panel design in which autoregresssive paths were specified for insomnia symptoms, SWB, EWB, and psychological distress, with insomnia symptoms at Time 1 being specified as a predictor of SWB, EWB, and psychological distress at Time 2. The third model was a longitudinal panel design similar to the second model, but specified recurring insomnia symptoms as a predictor of SWB, EWB, and psychological distress at Time 2. Age and gender were specified as covariates in each of the three models. To avoid estimation problems, we divided age by 10 to render its scale more comparable to that of other variables in the model. Physical illness score was included as a covariate in the first model, but was removed as a covariate in the second and third longitudinal models for statistical parsimony. The small amount of missing data (covariance coverage ranged from $96.4 \%$ to $100 \%$ ) was addressed by imputing

Table 2

Means, Standard Deviations, and Zero-Order Correlations Among Insomnia Symptoms, Well-Being, and Psychological Distress in MIDUS-II

\begin{tabular}{|c|c|c|c|c|c|c|c|c|c|c|c|c|c|}
\hline \multirow[b]{2}{*}{ Variable } & \multirow[b]{2}{*}{ INS } & \multicolumn{3}{|c|}{ SWB } & \multicolumn{6}{|c|}{ EWB } & \multicolumn{3}{|c|}{$\mathrm{PD}$} \\
\hline & & LS & NA & PA & $\mathrm{AU}$ & EM & PG & PR & PL & SA & DEP & GAD & PAN \\
\hline Mean & 3.92 & 7.88 & 4.49 & 3.43 & 37.14 & 38.19 & 38.42 & 40.58 & 38.43 & 38.15 & .60 & .11 & .30 \\
\hline Standard deviation & 1.76 & 1.53 & .58 & .70 & 6.95 & 7.39 & 6.92 & 6.96 & 6.98 & 8.19 & 1.71 & .79 & .98 \\
\hline Insomnia symptoms (INS) & - & & & & & & & & & & & & \\
\hline Life satisfaction (LS) & -.20 & - & & & & & & & & & & & \\
\hline Negative affect (NA) & -.34 & .46 & - & & & & & & & & & & \\
\hline Positive affect (PA) & -.28 & .56 & .60 & - & & & & & & & & & \\
\hline Autonomy (AU) & -.15 & .23 & .32 & .30 & - & & & & & & & & \\
\hline Environmental mastery (EM) & -.24 & .53 & .57 & .58 & .52 & - & & & & & & & \\
\hline Personal growth (PG) & -.18 & .39 & .38 & .41 & .43 & .58 & - & & & & & & \\
\hline Positive relations (PR) & -.16 & .43 & .39 & .46 & .38 & .62 & .58 & - & & & & & \\
\hline Purpose in life (PL) & -.19 & .44 & .43 & .44 & .40 & .63 & .69 & .60 & - & & & & \\
\hline Self acceptance (SA) & -.23 & .58 & .53 & .59 & .51 & .76 & .64 & .66 & .68 & - & & & \\
\hline Depression (DEP) & .20 & -.26 & -.44 & -.35 & -.13 & -.30 & -.16 & -.19 & -.21 & -.30 & - & & \\
\hline Generalized anxiety (GAD) & .13 & -.16 & -.30 & -.21 & -.11 & -.20 & -.12 & -.11 & -.13 & -.20 & .30 & - & \\
\hline Panic attacks (PAN) & .16 & -.14 & -.27 & -.20 & -.06 & -.18 & -.06 & -.09 & -.09 & -.17 & .27 & .15 & - \\
\hline
\end{tabular}

Note. $\mathrm{SWB}=$ subjective well-being; $\mathrm{EWB}=$ eudaimonic well-being; $\mathrm{PD}=$ psychological distress. All correlations are significant at the $p<.001$ level. 
data using PRELIS (Jöreskog \& Sörbom, 1996). The SEM models were then analyzed using maximum likelihood estimation procedures in LISREL 8.7 (Jöreskog \& Sörbom, 2006). Model fit was evaluated using four of the most commonly accepted indices of fit: root mean square error of approximation (RMSEA), nonnormed fit index (NNFI), comparative fit index (CFI), and the standardized root mean residual (SRMR). RMSEA and SRMR values ranging from .05 to .08 are considered "acceptable," .01-.05 are considered indicative of "close fit," and .00 would indicate a perfect or exact fit. NNFI and CFI are relative fit indices, with values of 0.90 to 0.95 reflecting acceptable fit, $0.95-0.99$ indicating close fit, and a value of 1.00 would indicate perfect fit.

\section{Results}

In the MIDUS-II sample, a large proportion $(27.5 \%, n=1,105)$ of this sample of middle-aged adults reported never experiencing insomnia symptoms, and $29.2 \%(n=1,174)$ reported experiencing insomnia symptoms several times per week or almost every night (see Table 1). Consistent with other research, in the MIDUS-II sample, there was a small but statistically significant relationship between age and insomnia symptoms $(r=.05, p<.005)$, as well as gender and insomnia symptoms, $t(3997)=-6.77, p<.001$, such that older adults and women reported more frequent insomnia symptoms than younger adults and men. There was also a positive association between physical illness severity and insomnia symptoms $(r=.15, p<.001)$. Furthermore, as seen in Table 2 , in the MIDUS-II sample, insomnia symptoms were significantly associated with decreased SWB, decreased EWB, and increased psychological distress. Regarding recurrent insomnia symptoms, approximately half $(n=2,065)$ of participants reported never experiencing insomnia symptoms during the past 30 days at one or both time points, whereas $10.0 \%(n=402)$ of participants reported experiencing insomnia symptoms several times per week or almost every night at both Time 1 and Time 2 (see Table 1).

\section{Model 1: Insomnia Symptoms, Well-Being, and Psychological Distress in MIDUS-II}

Our first model specified insomnia symptoms as associated with the latent variables SWB, EWB, and psychological distress, while covarying for the effects of age, gender, and physical illness score. The completely standardized results of this model can be seen in Figure 1. The overall model fit was good, $\chi^{2}(48, N=4,014)=$ 1003.76, NNFI $=0.94, \mathrm{CFI}=0.97, \mathrm{RMSEA}=.07, \mathrm{SRMR}=$ .035. Replicating our previous findings, the negative association of insomnia symptoms with SWB was significant, $B=-.401, S E=$ $.020, z=20.08$, along with the negative association of insomnia symptoms with EWB, $B=-.241, S E=.017, z=14.01$. Extending our previous work, results also indicated that insomnia symptoms were related to increased psychological distress, $B=.315$, $S E=.024, z=12.94$.

\section{Model 2: Longitudinal Effects of Insomnia Symptoms on Well-Being and Psychological Distress}

The second model examined whether insomnia symptoms at Time 1 predicted well-being and psychological distress at 10-year follow-up (Time 2). A longitudinal path model was specified in

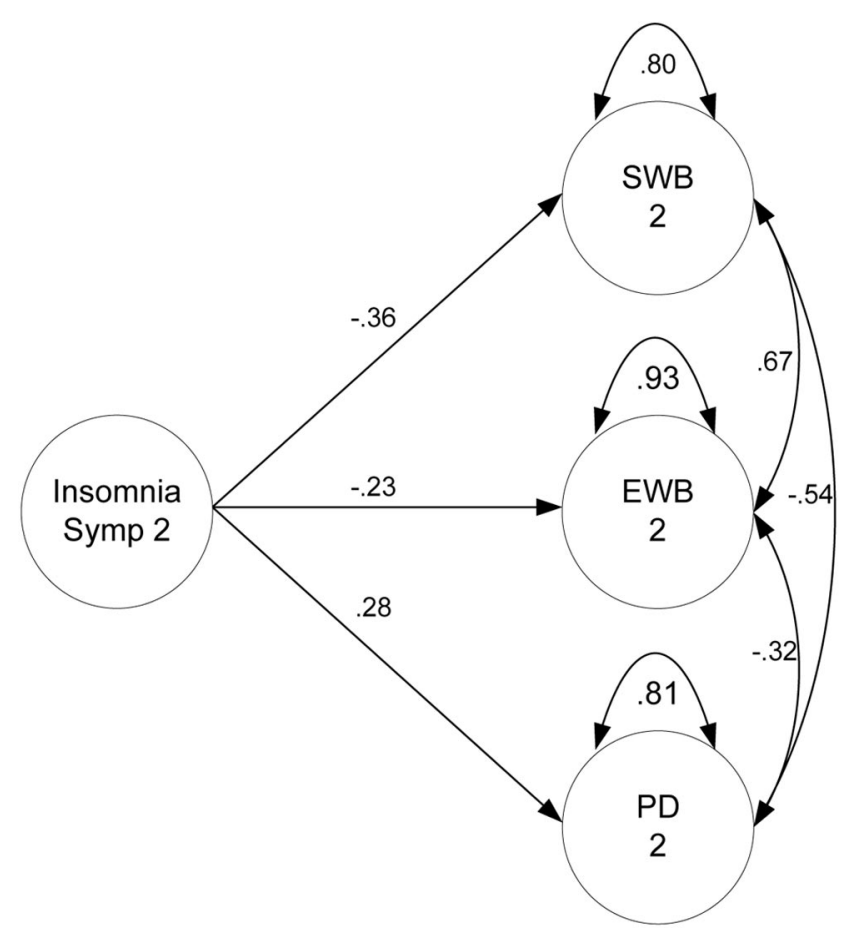

Figure 1. Insomnia symptoms, well-being, and psychological distress in MIDUS-II. This figure illustrates a model in which the effects of insomnia symptoms on SWB, EWB, and psychological distress are estimated after adjusting for age, gender, and physical illness score. All path coefficients represent completely standardized values. All effects displayed are statistically significant at $p<05$. Insomnia Symp = insomnia symptoms; SWB = subjective well-being; EWB = eudaimonic well-being; $\mathrm{PD}=$ psychological distress.

which autoregressive paths were defined for SWB, EWB, and psychological distress, while covarying for the effects of age and gender. Insomnia symptoms at Time 1 were specified as a correlate of Time 1 outcomes and as a predictor of Time 2 outcomes. The completely standardized results of this model can be seen in Figure 2. The overall model fit was good, $\chi^{2}(168, N=4,014)=1801.79$, $\mathrm{NNFI}=0.97, \mathrm{CFI}=0.98, \mathrm{RMSEA}=.05, \mathrm{SRMR}=.038$ Because we were interested in the degree to which insomnia symptoms at Time 1 predicted future well-being and psychological distress, we examined regression weights for each of these relationships. As can be seen in Figure 2, insomnia symptoms at Time 1 were not found to be a significant predictor of future SWB, $B=$ $.007, S E=.017, z=0.41$, or EWB, $B=-.023, S E=.014, z=$ -1.7 . However, insomnia at Time 1 was found to predict psychological distress at 10-year follow-up, $B=.07, S E=.02, z=2.9$. This effect existed even while controlling for age, gender, and Time 1 level of psychological distress.

\section{Model 3: Longitudinal Effects of Recurring Insomnia Symptoms on Well-Being and Psychological Distress}

The third model examined whether a participant's minimum frequency of insomnia symptoms at Time 1 and Time 2 (i.e., recurring insomnia symptoms score) functioned as a vulnerability factor for lower levels of well-being and higher levels of psycho- 


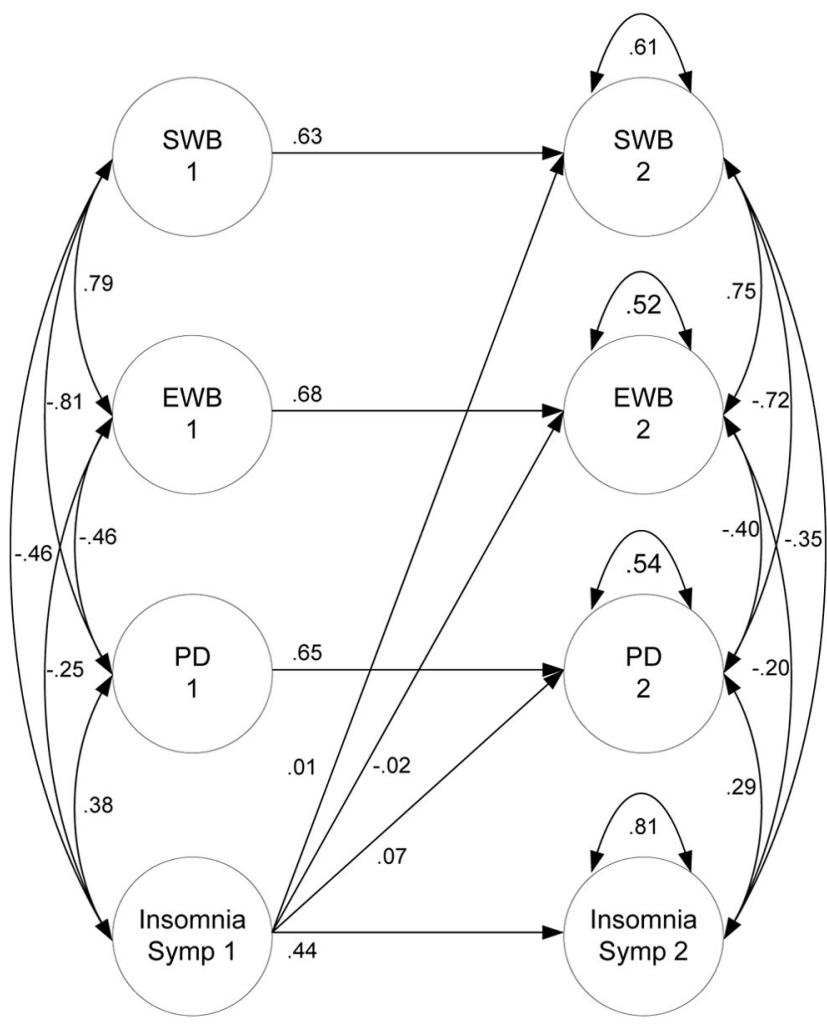

Figure 2. Longitudinal effects of insomnia symptoms on well-being and psychological distress. This figure illustrates a model in which the longitudinal effects of insomnia symptoms on SWB, EWB, and psychological distress are estimated after adjusting for age and gender. All path coefficients represent completely standardized values. All effects greater than .06 are statistically significant. Insomnia Symp = insomnia symptoms; SWB = subjective well-being; EWB = eudaimonic well-being; $\mathrm{PD}=$ psychological distress.

logical distress. A longitudinal panel model was specified in which autoregressive paths were defined for SWB, EWB, and psychological distress, while covarying for the effects of age and gender. Recurring insomnia symptoms were specified as a correlate of Time 1 outcomes and as a predictor of Time 2 outcomes. The completely standardized results of this model can be seen in Figure 3 . The overall model fit was good, $\chi^{2}(162, N=4,014)=4070.55$, $\mathrm{NNFI}=0.93, \mathrm{CFI}=0.94, \mathrm{RMSEA}=.08, \mathrm{SRMR}=.044$. As can be seen in Figure 3, recurring insomnia symptoms had a significant negative association with $\mathrm{SWB}, B=-.232, S E=.023, z=10.19$; a significant negative association with EWB, $B=-.129, S E=$ $.020, z=6.35$; and a significant positive association with psychological distress, $B=.257, S E=.038, z=6.73$. These effects existed while controlling for age, gender, and Time 1 well-being and psychological distress.

Supplemental Cohen's $d$ effect size analyses were conducted next to further examine the effect of recurring clinically frequent insomnia symptoms on well-being and psychological distress at Time 2. Clinically frequent insomnia symptoms were defined as participant-reported trouble sleeping several nights a week or almost every night at both Time 1 and Time 2. In support of our primary analyses, and as shown in Table 3 , there was a moderate to large effect size of recurring clinically frequent insomnia symptoms on SWB, EWB, and psychological distress in comparison to participants who experienced clinically frequent insomnia symptoms at only one time point or did not experience clinically frequent insomnia symptoms at either time point (i.e., nonrecurring clinically frequent insomnia symptoms).

\section{Discussion}

Results of this study suggest that the experience of recurring insomnia symptoms at two time points 10 years apart is predictive of decreased well-being and increased psychological distress. In this study, we first replicated our previous findings (Hamilton et al., 2007) that concurrent insomnia symptoms may play an important role in determining an individual's levels of SWB and EWB. These findings are consistent with the few other studies that have investigated the role of sleep on psychological well-being, which found that sleep disturbance was related to decreased psychological well-being in mothers of children with developmental disabilities (Chu \& Richdale, 2009) and good sleep quality was related to increased psychological well-being in Japanese working men (Kawada et al., 2011). In this study, Model 1 also expanded our

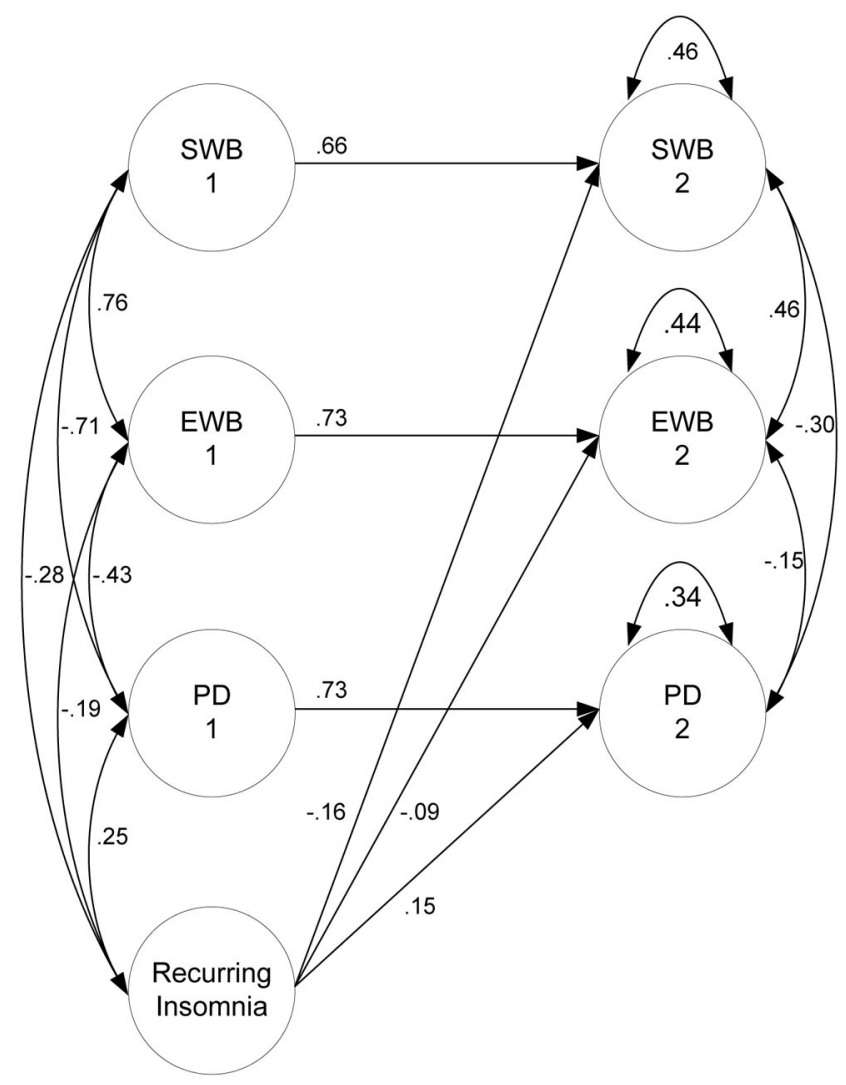

Figure 3. Longitudinal effects of recurring insomnia symptoms on wellbeing and psychological distress. This figure illustrates a model in which the longitudinal effects of recurring insomnia symptoms on SWB, EWB, and psychological distress are estimated after adjusting for age and gender. All path coefficients represent completely standardized values. All effects greater than .02 are statistically significant. SWB = subjective well-being; $\mathrm{EWB}=$ eudaimonic well-being; $\mathrm{PD}=$ psychological distress. 
Table 3

Effect Sizes (Cohen's d) for Recurring Clinically Frequent Insomnia Symptoms on Well-Being and Psychological Distress in MIDUS-II

\begin{tabular}{|c|c|c|c|c|c|c|c|}
\hline \multirow[b]{2}{*}{ Outcome variable } & \multicolumn{3}{|c|}{$\begin{array}{l}\text { Nonrecurring clinically frequent } \\
\text { insomnia symptoms }\end{array}$} & \multicolumn{3}{|c|}{$\begin{array}{l}\text { Recurring clinically frequent } \\
\text { insomnia symptoms }\end{array}$} & \multirow[b]{2}{*}{ Cohen's $d(95 \%$ CI $)$} \\
\hline & $n$ & $M$ & $S D$ & $n$ & $M$ & $S D$ & \\
\hline \multicolumn{8}{|l|}{ SWB } \\
\hline Life satisfaction & 3,472 & 7.95 & 1.47 & 400 & 7.26 & 1.88 & $-0.45[-0.35,-0.56]$ \\
\hline Negative affect & 3,490 & 4.53 & 0.53 & 396 & 4.10 & 0.76 & $-0.77[-0.67,-0.88]$ \\
\hline Positive affect & 3,508 & 3.47 & 0.68 & 398 & 3.05 & 0.79 & $-0.61[-0.50,-0.71]$ \\
\hline \multicolumn{8}{|l|}{ EWB } \\
\hline Autonomy & 3,507 & 37.34 & 6.86 & 400 & 35.51 & 7.42 & $-0.27[-0.16,-0.37]$ \\
\hline Environmental mastery & 3,507 & 38.59 & 7.23 & 400 & 34.70 & 7.97 & $-0.53[-0.43,-0.64]$ \\
\hline Personal growth & 3,507 & 38.69 & 6.80 & 400 & 35.95 & 7.53 & $-0.40[-0.29,-0.50]$ \\
\hline Positive relations & 3,507 & 40.81 & 6.86 & 400 & 38.51 & 7.58 & $-0.33[-0.23,-0.43]$ \\
\hline Purpose in life & 3,507 & 38.77 & 6.86 & 400 & 35.61 & 7.30 & $-0.46[-0.35,-0.56]$ \\
\hline Self-acceptance & 3,507 & 38.59 & 7.97 & 400 & 34.30 & 9.16 & $-0.53[-0.43,-0.63]$ \\
\hline \multicolumn{8}{|l|}{ Psychological distress } \\
\hline Depression & 3,519 & 0.50 & 1.55 & 402 & 1.49 & 2.57 & $0.59[0.69,0.48]$ \\
\hline Generalized anxiety & 3,519 & 0.07 & 0.59 & 402 & 0.46 & 1.64 & $0.51[0.61,0.41]$ \\
\hline Panic attacks & 3,519 & 0.25 & 0.88 & 402 & 0.73 & 1.53 & $0.50[0.60,0.39]$ \\
\hline
\end{tabular}

Note. $\quad \mathrm{SWB}=$ subjective well-being; EWB = eudaimonic well-being. Negative effect sizes indicate that recurring clinically frequent insomnia symptoms were associated with lower levels of an outcome, and positive effect sizes indicate increased levels of an outcome. Negative affect items were reverse coded so that a positive directional relationship indicated better well-being.

previous findings by using a large national sample to replicate other research (e.g., Kokras et al., 2011; Morphy, Dunn, Lewis, Boardman, \& Croft, 2007) that suggests that concurrent insomnia symptoms are related to increased psychological distress.

In our second study model, we found that insomnia symptoms at Time 1 were not a significant predictor of future SWB or EWB. However, consistent with past research (Ford \& Kamerow, 1989; Yokoyama et al., 2010), insomnia symptoms at Time 1 were found to predict psychological distress at 10-year follow-up. In addition, insomnia symptoms at Time 1 were significantly associated with insomnia symptoms at Time 2. Leading from these findings, whereas insomnia symptoms at Time 1 were not predictive of well-being at Time 2, in Model 3, recurring insomnia symptoms at Time 1 and Time 2 were found to be significantly related to decreased SWB and EWB, as well as increased psychological distress. Model 3 findings were further supported in supplemental analyses that indicated that participants who experienced clinically frequent insomnia symptoms (i.e., trouble sleeping several nights a week or almost every night) at both Time 1 and Time 2 reported lower SWB, lower EWB, and higher psychological distress at Time 2 than did participants who did not experience recurring clinically frequent insomnia symptoms.

These results suggest that insomnia symptoms assessed at a single time point may not be predictive of future psychological well-being, but that insomnia symptoms at a single time point may serve as a risk factor for future symptoms of psychological distress, as well as future symptoms of insomnia. Results further suggest that the recurring experience of insomnia symptoms, even 10 years apart, leaves an individual vulnerable to increased psychological distress and decreased well-being. Recurring insomnia symptoms appear to negatively impact many dimensions of wellbeing, perhaps most significantly an individual's mood, sense of environmental mastery, and self-acceptance. With recurring in- somnia symptoms, individuals may feel less in control of their environment and less accepting of their current functioning in life. This apparent increased vulnerability may have significant implications for the importance of treating insomnia symptoms and sleep disturbance early on, particularly in individuals who experience insomnia symptoms in combination with psychological illnesses such as depression.

Insomnia symptoms and sleep disturbance are common to many somatic illnesses as well as psychological illnesses such as depression and anxiety (Dikeos \& Georgantopoulos, 2011; Karlson, Stevens, Olson, \& Hamilton, 2010; Sevim et al., 2004). In fact, longitudinal research suggests that insomnia symptoms may portent future depressive symptoms and depressive episodes (Baglioni et al., 2011; Demyttenaere, De Fruyt, \& Stahl, 2005). Such research findings are consistent with the theory of allostatic load, which conceptualizes an individual's attempt to adapt to life's demands as a cumulative physiological burden exacted on the body (McEwen, 1998). Thus, individuals who experience insomnia symptoms may experience a heightened stress response and decreased ability to attend to, engage in, or enjoy pleasurable activities (Hamilton et al., 2008; Zohar, Tzischinsky, Epstein, \& Lavie, 2005), making them vulnerable to decreased well-being and increased psychological distress. It therefore may be the case that individuals who experience recurring insomnia symptoms, even in isolation, would benefit from behavioral treatments such as cognitive-behavioral therapy for insomnia to reduce future sleep disturbance and thereby reduce their risk for poorer psychological health and well-being outcomes.

Despite the statistical strengths of this study (e.g., the use of SEM to model the effects of insomnia symptoms on the longitudinal course of well-being and psychological distress), there are several study limitations. The primary limitation is the reliance on a single retrospective question to assess the frequency of insomnia 
symptoms. Although other epidemiological studies that have linked sleep disturbance to mortality have also relied on a single question to identify the presence of sleep disturbance and severity of insomnia (e.g., Chen et al., 2008; Kripke, Garfinkel, Wingard, Klauber, \& Marler, 2002), future studies would advance current study findings by including an expanded question set as well as objective measures of sleep disturbance (e.g., actigraphy). Furthermore, although it has been established that the perception of poor sleep is important to the experience of insomnia regardless of actual sleep (e.g., Fernandez-Mendoza et al., 2011), and commonly used sleep questionnaires such as the Pittsburg Sleep Quality Index (Buysse, Reynolds, Monk, Berman, \& Kupfer, 1989) are retrospective in nature, future studies should make attempts to employ additional DSM criteria to establish a diagnosis of insomnia. In addition, similar studies would benefit from assessing other sleep disorders such as obstructive sleep apnea, narcolepsy, and restless leg syndrome given that insomnia symptoms may be related to other sleep disorders.

Another limitation of this study is that insomnia symptoms, well-being, and psychological distress were measured only at two time points 10 years apart from a somewhat homogeneous, primarily White population. Although the study population was a large sample of adults across the United States, spanning a wide age range, no data were collected from participants regarding sleep or well-being during the time between MIDUS-I and MIDUS-II. Therefore, it is not known whether symptoms of insomnia experienced at these two time points were singular events or whether they captured ongoing symptoms of insomnia. Future studies examining the impact of recurring insomnia symptoms on well-being and psychological distress should obtain more frequent measures of these variables in a more diverse population.

In conclusion, this study supports previous findings that insomnia symptoms may play an important role in determining an individual's current psychological well-being and future psychological distress. Furthermore, study results extend this body of research to suggest that recurring insomnia symptoms may endow a particular vulnerability to decreased psychological well-being and increased psychological distress. Results of this study support a growing body of literature that emphasizes the importance of adequate sleep in psychological well-being and health (e.g., Cukrowicz, Smith, Hohmeister, \& Joiner, 2009).

\section{References}

Adam, K., \& Oswald, I. (1977). Sleep is for tissue restoration. Journal of the Royal College of Physicians of London, 11, 376-388.

American Psychiatric Association. (1987). Diagnostic and statistical manual of mental disorders (3rd ed. rev.). Washington, DC: Author.

Baglioni, C., Battagliese, G., Feige, B., Spiegelhalder, K., Nissen, C., Voderholzer, U., ... Riemann, D. (2011). Insomnia as a predictor of depression: A meta-analytic evaluation of longitudinal epidemiological studies. Journal of Affective Disorders, 135, 10-19. doi:10.1016/ j.jad.2011.01.011

Beekman, A. T., Penninx, B. W., Deeg, D. J., de Beurs, E., Geerling, S. W., \& van Tilburg, W. (2002). The impact of depression on the well-being, disability and use of services in older adults: A longitudinal perspective. Acta Psychiatrica Scandinavica, 105, 20-27. doi:10.1034/j.16000447.2002.10078.x

Benyamini, Y., Leventhal, E. A., \& Leventhal, H. (1999). Self-assessments of health: What do people know that predicts their mortality? Research on Aging, 21, 477-500. doi:10.1177/0164027599213007
Bixler, E. O., Vgontzas, A. N., Lin, H. M., Vela-Bueno, A., \& Kales, A. (2002). Insomnia in central Pennsylvania. Journal of Psychosomatic Research, 53, 589-592. doi:10.1016/S0022-3999(02)00450-6

Breslau, N., Roth, T., Rosenthal, L., \& Andreski, P. (1996). Sleep disturbance and psychiatric disorders: A longitudinal epidemiological study of young adults. Biological Psychiatry, 39, 411-418. doi:10.1016/00063223(95)00188-3

Buysse, D. J., Reynolds, C. F., Monk, T. H., Berman, S. R., \& Kupfer, D. J. (1989). The Pittsburgh Sleep Quality Index (PSQI): A new instrument for psychiatric research and practice. Psychiatry Research, 28, 193-213. doi:10.1016/0165-1781(89)90047-4

Cantril, H. (1965). The pattern of human concerns. New Brunswick, NJ: Rutgers University Press.

Carli, V., Roy, A., Bevilacqua, L., Maggi, S., Cesaro, C., \& Sarchiapone, M. (2011). Insomnia and suicidal behaviour in prisoners. Psychiatry Research, 185, 141-144. doi:10.1016/j.psychres.2009.10.001

Chen, J. C., Brunner, R. L., Ren, H., Wassertheil-Smoller, S., Larson, J. C., Levine, D. W., .. Stefanick, M. L. (2008). Sleep duration and risk of ischemic stroke in postmenopausal women. Stroke, 39, 3185-3192. doi:10.1161/STROKEAHA.108.521773

Chu, J., \& Richdale, A. L. (2009). Sleep quality and psychological wellbeing in mothers of children with developmental disabilities. Research in Developmental Disabilities, 30, 1512-1522. doi:10.1016/ j.ridd.2009.07.007

Cukrowicz, K. C., Smith, P. N., Hohmeister, H. C., \& Joiner, T. E., Jr. (2009). The moderation of an early intervention program for anxiety and depression by specific psychological symptoms. Journal of Clinical Psychology, 65, 337-351. doi:10.1002/jclp.20560

Demyttenaere, K., De Fruyt, J., \& Stahl, S. M. (2005). The many faces of fatigue in major depressive disorder. International Journal of Neuropsychopharmacology, 8, 93-105. doi:10.1017/S1461145704004729

Dikeos, D., \& Georgantopoulos, G. (2011). Medical comorbidity of sleep disorders. Current Opinion in Psychiatry, 24, 346-354. doi:10.1097/ YCO.0b013e3283473375

Fernandez-Mendoza, J., Calhoun, S. L., Bixler, E. O., Karataraki, M., Liao, D., Vela-Bueno, A., ... Vgontzas, A. N. (2011). Sleep misperception and chronic insomnia in the general population: Role of objective sleep duration and psychological profiles. Psychosomatic Medicine, 73, 8897. doi:10.1097/PSY.0b013e3181fe365a

Foley, D., Ancoli-Israel, S., Britz, P., \& Walsh, J. (2004). Sleep disturbances and chronic disease in older adults: Results of the 2003 National Sleep Foundation Sleep in America Survey. Journal of Psychosomatic Research, 56, 497-502. doi:10.1016/j.jpsychores.2004.02.010

Ford, D. E., \& Kamerow, D. B. (1989). Epidemiologic study of sleep disturbances and psychiatric disorders. Journal of the American Medical Association, 262, 1479-1484. doi:10.1001/jama.1989.03430110069030

Hamilton, N. A., Affleck, G., Tennen, H., Karlson, C., Luxton, D., Preacher, K. J., \& Templin, J. L. (2008). Fibromyalgia: The role of sleep and affect in negative event reactivity and recovery. Health Psychology, 27, 490-497. doi:10.1037/0278-6133.27.4.490

Hamilton, N. A., Gallagher, M., Preacher, C., Stevens, N., Nelson, C. A., Karlson, C., \& McCurdy, D. (2007). Insomnia and well-being. Journal of Consulting and Clinical Psychology, 75, 939-946. doi:10.1037/0022006X.75.6.939

Jöreskog, K. G., \& Sörbom, D. (1996). LISREL 8 user's reference guide. Uppsala, Sweden: Scientific Software International.

Jöreskog, K. G., \& Sörbom, D. (2006). LISREL for Windows [Computer software]. Lincolnwood, IL: Scientific Software International, Inc.

Karlson, C. W., Stevens, N. R., Olson, C. A., \& Hamilton, N. A. (2010). Depression, fatigue, and pre-sleep arousal: A mediational model. Journal of College Student Psychotherapy, 24, 307-327. doi:10.1080/ 87568225.2010.509259

Kawada, T., Otsuka, T., Inagaki, H., Wakayama, Y., Katsumata, M., Li, Q., \& Li, Y.-J. (2011). Relationship among lifestyles, aging and psycholog- 
ical wellbeing using the General Health Questionnaire 12-items in Japanese working men. Aging Male, 14, 115-118. doi:10.3109/ 13685538.2010.493588

Kessler, R. C., Andrews, A., Mroczek, D., Ustun, B., \& Wittchen, H. U. (1998). The World Health Organization Composite International Diagnostic Interview-Short Form (CIDI-SF). International Journal of Methods in Psychiatric Research, 7, 171-185. doi:10.1002/mpr.47

Keyes, C. L. M., Shmotkin, D., \& Ryff, C. D. (2002). Optimizing wellbeing: The empirical encounter of two traditions. Journal of Personality and Social Psychology, 82, 1007-1022. doi:10.1037/00223514.82.6.1007

Kokras, N., Kouzoupis, A. V., Paparrigopoulos, T., Ferentinos, P., Karamanakos, P., \& Kontoyannis, D. A. (2011). Predicting insomnia in medical wards: The effect of anxiety, depression and admission diagnosis. General Hospital Psychiatry, 33, 78-81. doi:10.1016/ j.genhosppsych.2010.12.003

Kripke, D., Garfinkel, L., Wingard, D., Klauber, M., \& Marler, M. (2002). Mortality associated with sleep duration and insomnia. Archives of General Psychiatry, 59, 131-136. doi:10.1001/archpsyc.59.2.131

Léger, D., Scheuermaier, K., Philip, P., Paillard, M., \& Guilleminault, C. (2001). SF-36: Evaluation of quality of life in severe and mild insomniacs compared with good sleepers. Psychosomatic Medicine, $63,49-55$.

Little, T. D., Cunningham, W., Shahar, G., \& Widaman, K. (2002). To parcel or not to parcel: Exploring the question, weighing the merits. Structural Equation Modeling, 9, 151-173. doi:10.1207/ S15328007SEM0902_1

McEwen, B. S. (1998). Protective and damaging effects of stress mediators. New England Journal of Medicine, 338, 171-179. doi:10.1056/ NEJM199801153380307

Morphy, H., Dunn, K. M., Lewis, M., Boardman, H. F., \& Croft, P. R. (2007). Epidemiology of insomnia: A longitudinal study in a UK population. Sleep, 30, 274-280.

Mroczek, D. K., \& Kolarz, C. M. (1998). The effect of age on positive and negative affect: A developmental perspective on happiness. Journal of Personality and Social Psychology, 75, 5, 1333-1349. doi:10.1037/ 0022-3514.75.5.1333

Nissen, C., Kloepfer, C., Feige, B., Piosczyk, H., Spiegelhalder, K., Voderholzer, U., \& Riemann, D. (2011). Sleep-related memory consolidation in primary insomnia. Journal of Sleep Research, 20, 129-136. doi:10.1111/j.1365-2869.2010.00872.x

Ohayon, M. M. (2005). Relationship between chronic painful physical condition and insomnia. Journal of Psychiatric Research, 39, 151-159. doi:10.1016/j.jpsychires.2004.07.001
Philip, P., Léger, D., Taillard, J., Quera-Salva, M. A., Niedhammer, I., Mosqueda, J. G., . . Gérard, D. (2006). Insomniac complaints interfere with quality of life but not with absenteeism: Respective role of depressive and organic comorbidity. Sleep Medicine, 7, 585-591. doi:10.1016/ j.sleep.2006.04.006

Redeker, N. S., Jeon, S., Muench, U., Campbell, D., Walsleben, J., \& Rapoport, D. M. (2010). Insomnia symptoms and daytime function in stable heart failure. Sleep, 33, 1210-1216.

Ryff, C. D. (1989). Happiness is everything, or is it? Explorations on the meaning of psychological well-being. Journal of Personality and Social Psychology, 57, 1069-1081. doi:10.1037/0022-3514.57.6.1069

Ryff, C. D., \& Singer, B. (1996). Psychological well-being: Meaning, measurement, and implications for psychotherapy research. Psychotherapy and Psychosomatics, 65, 14-23. doi:10.1159/000289026

Sevim, S., Dogu, O., Kaleagasi, H., Aral, M., Metin, O., \& Camdeviren, H (2004). Correlation of anxiety and depression symptoms in patients with restless legs syndrome: A population based survey. Journal of Neurology, Neurosurgery \& Psychiatry, 75, 226-230.

Steptoe, A., O'Donnell, K., Marmot, M., \& Wardle, J. (2008). Positive affect, psychological well-being, and good sleep. Journal of Psychosomatic Research, 64, 409-415. doi:10.1016/j.jpsychores.2007.11.008

Uchino, B. N., Cacioppo, J. T., \& Kiecolt-Glaser, J. K. (1996). The relationship between social support and physiological processes: A review with emphasis on underlying mechanisms and implications for health. Psychological Bulletin, 119, 488-531. doi:10.1037/00332909.119.3.488

Wittchen, H. U. (1994). Reliability and validity studies of the WHOComposite International Diagnostic Interview: A critical review. Journal of Psychiatric Research, 28, 57-84. doi:10.1016/0022-3956(94)90036-1

Yokoyama, E., Kaneita, Y., Saito, Y., Uchiyama, M., Matsuzaki, Y., Tamaki, T., ... Ohida, T. (2010). Association between depression and insomnia subtypes: A longitudinal study on the elderly in Japan. Sleep, 33, 1693-1702.

Zammit, G. K., Weiner, J., Damato, N., Sillup, G. P., \& McMillan, C. A. (1999). Quality of life in people with insomnia. Sleep, 22, S379-S385.

Zohar, D., Tzischinsky, O., Epstein, R., \& Lavie, P. (2005). The effects of sleep loss on medical residents' emotional reactions to work events: A cognitive-energy model. Sleep, 28, 47-54

Received July 27, 2011

Revision received January 23, 2012

Accepted January 25, 2012 